\title{
Use of Added Damping Devices with Special Emphasis on Multiple Tuned Mass Dampers : A Review \\ Avinash Kalamkar ${ }^{1}$, N. H. Pitale², P. B. Patil ${ }^{3}$ \\ 1PG Scholar, Structural Engineering, G.H. Raisoni College of Engineering, Nagpur, Maharashtra, India. \\ ${ }^{2,3}$ Assistant Professor, Civil Engineering, G.H. Raisoni College of Engineering, Nagpur, Maharashtra, India
}

\section{ABSTRACT}

Article Info

Volume 8, Issue 1

Page Number: 227-235

Publication Issue :

January-February-2021

Article History

Accepted : 08 Feb 2021

Published : 18 Feb 2021
Dampers are significant part of structure in the highlight increment seismic opposition. As they increment the life of structure, toughness and flexiblity. In various power condition the conduct of damper assumes a significant job, which shows the need of damper.So it is important to concentrate to make its utilization in better manner.

Keywords : MTMD ( Multiple Tuned Mass Dampers), supplemental damping, hysteresis.

\section{INTRODUCTION}

Dampers play an important role in structure. There are different types of dampers, behavior of these connection changes as per condition. We will study the behavior of connection in thermal conditions. Hybrid connection, straight of it. Here the various aspects of structure are studied under the effect of dampers. Comparison of different types of dampers is done to see which is better. There are several issues with systems and machines created by humans due to vibrations. Vibration in a building or a car can be distracting or may lead to metal fatigue or a structural failure. The most effective and cheapest way to minimize vibration issues in many circumstances is to implement damping, the vibration energy reduction process.

\section{LITERATURE REVIEW}

[1] Kenneth O'Neill, P.E., Erik Bishop, P.E., David Swanson, P.E., S.E., Bradley Martin, P.E., S.E., and Drew Nielson:- In the thesis the authors focused on non-structural research of the two pre-Northridge steel moment frame-buildings on the west coast of Switzerland. Function on the basis of code equations ASCE 7 and ASCE 41, the study involves a less strict estimate. The damper was additional damper here used for the design purpose. The spectral acceleration of the non-linear reaction background study of ground acceleration was measured in this Nonstructural part. In measuring the SDOF elastic oscillator response the results obtained from the dynamic amplification factor can be much higher than the $2.5 \%$ flexible amplification factor recommended in ASCE 7 and in ASCE 41 according to the period of the component and the assumptions made for study. Many analytical experiments found

Copyright: (C) the author(s), publisher and licensee Technoscience Academy. This is an open-access article distributed under the terms of the Creative Commons Attribution Non-Commercial License, which permits unrestricted noncommercial use, distribution, and reproduction in any medium, provided the original work is properly cited 
that the value of the ap code could be too little, but many test results suggest that the value of the ap code is appropriate. Horizontal acceleration equation and consequent horizontal design intensity for a non-

structural component

$$
F_{p}=\frac{0.4 a_{p} s_{X s} W_{p}\left(1+2 \frac{z}{h}\right)}{\left(\frac{R p}{I_{p}}\right)}
$$

[2] Hao Gao and Junjie Wang:-The authors explored differnces in the bi-directional seismic regulation between cylindrical and e-shaped dampers. For the purpose of minimizing seismic forces, metal dampers in cylinder and e-shapes were used. This dumping devices are tested for mechanical model parameters, hysteresis capacities, load patterns and test phenomena, material quantity failure modes and space specifications for installation and damping effects.

The results of this test revealed that during the test, eshaped dampers were effective and easy to attach to the plates. The E and C-shaped dampers with on-theground deformations need fewer installation and work space in comparative with cylindrical dampers, which makes them easier to handle with medium spanning and continuous girder bridges.

[3] Georgios Apostolakis, - The seismic optimum evolution of 3D multi-story buildings with damping devices has been researched. A computer system was developed for the seismic design of normal and unusual multi-story 3D frameworks. A 3D 8-story building with an interval steel resistant frame and a 14-story building with friction damper were designed and retrofitted. Through testing those two models, damping equipment was standardized over the entire structural height by introducing modern mega-brace three-dimensional architectures. For the seismic design and the refurbishment of realistic 3D buildings with damping systems, the proposed computational architecture offers an appealing option. In the reach of technical Architecture organizations, the system includes moderate programming and engineering.

[4] Mohammad Seddiq Eskandari and Jinkoo Kim:The consolidation of steel-cut and viscoelastic dampers associated with enhanced seismic execution of the structures has identified HSVD in this investigation. To accept energy diffusion capacities and the investigative model developed using the nonlinear BWBN model, cyclic stacking experiments were performed for both the viscoelastic damper and cross breed damper. The SAC Phase II business found a third-story second opposed edge and the logic model for seismic modifications was used for the assessment of the feasibility of HSVDs. Six implementation tests were used to analyze the structural reactions of seismic modifications with steel-cut dampers and HSVDs to obtain insight into the show of cross breed dampers. The Delicacy Analysis was then performed in order to examine the possibility of exceeding the breakpoints determined. In conclusion, the solution to the limit and request map was used to determine the need for equivalent seismic damping in order to satisfy the objective output argument in question.

After the structure was remodeled with HSVDs, the installation of optical dampers in the respective steel cutting dampers increased the overall appearance of the steel cuts. The findings of this analysis revealed The BWBN model was also found to compete better with the test results than the standard Kelvin-Voigt model. The delicacy study showed that the likelihood of arriving in dangerous states decreased as far as possible, particularly after seismic retrofitting with HSVDs. It's already been shown For small to medium tremors, HSVDs may be better. In the long term, the implementation of a cap and the request map technique are feasible in the fundamental plan to achieve the optimal target execution point of the cross race dampers. 


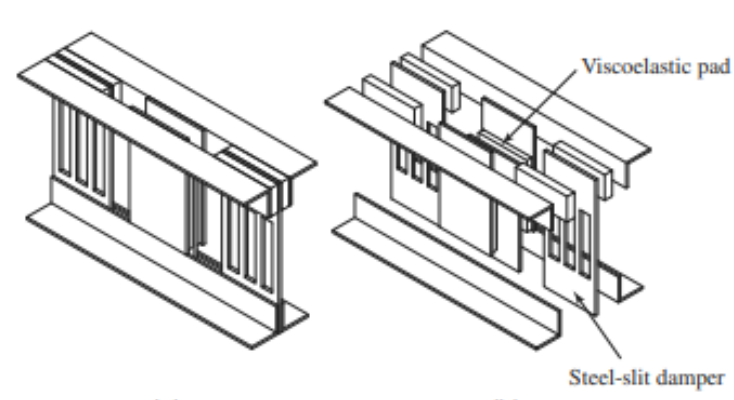

HSVD configuration: (a) hybrid damper; and (b) damper components.

[5] The author has worked in California on damping proportion analyzes in high constructions, taken from movements recorded in instruments during seismic shaking movements. Cristian Cruz and Eduardo Miranda: The minimum squared parametric context for identifiable proof is used to dissect 41 measured seismic reactions of 14 buildings of at least 20 stories. The efficiency of the damping measurements built is first measured in modular damping proportions based on the efficacy of the primary response on variants.

The damping ratios are observed to decrease, with increasing building height and damping levels are higher in solid structures than in steel structures, based on built damping proportions called solid. In addition, with little attention paid to the stuff, it has been found that the damping proportions of all structures above $150 \mathrm{~m}$ are lower than the seismic average of 2.5 per cent.

[6] "The authors analyzed the wind reaction of large buildings on flexible bases using many tuned mass dampers in this article. Elias \& Vasant Matsagar said (MTMDs). Here, because of soil-structure contact, MTMDs are positioned on top of the reinforced concrete frameworks (RC) (SSI). The solid circular foundation as well as the surrounding field are modeled on individual spring and dashpots frequency constants. For a non-classically damped device the time domain wind analysis based on an average acceleration process of Newmark is used. Compared to a single tuned mass damper, the output of the MTMDs is (STMD). In order to study the efficacy of the STMD and MTMD systems, the displacement and acceleration reaction in the wind and wind power tops of the structure. The findings concluded that the soil type has had an impact on the STMD and MTMD structural parameters and the building's wind reaction on the versatile floor. Furthermore, MTMDs are more powerful than STMDs.

[7] The authors in this research worked on Hysteretic Properties of Form Damper Devices (SDs). They were vertically arranged in frames with connecting beams in length, increasingly used in practice in timeresistance (SMRF) systems. In this analysis, the authors worked on the hysteretic characteristics of experiments. This damping devices are used to increase structural rigidity and energy dissipation to enhance seismical efficiency while retaining good structural architectural operation. It also provides an overview that clarifies the advantages of implementing SDFs in relation to their counterpart SMRFs and their impact on seismic efficiency at various levels of seismic risk from any hysteretic property of SDs. A collection of hyperetic properties of SDs has been proposed and reviewed to boost seismic performance in small and frequent earthquakes significantly. A set of hysteretic properties of the SDs is developed and tested to significantly improve seismic performance of the system under small and frequent earthquakes and to efficiently minimize waste. A tool to determine potential SDF residual plot drifts based on the ductility of the story has also been established..

[8] Mahmoud Kamalzar, Erik A. Johnson, Steven F. Wojtkiewicz,:- This paper recommends the use of an inputs for controllable DMGs based on the computationally efficient leisure strategy. The paper first summarizes the overall leisure approach for direct structures accompanied by brief clarification of three standard versions, modified and modular reduction techniques, Guyana. At this point, a worldview is given for the description of the controls, and depending on the reduced demand model of the structure, the KF spectator fuse is generated. Two 
mathematical models, a modell for a compact request system and a 3D high-order construction model are used for evaluation of the technique. The damping goals and imperatives are seen with each model, model, enthusiasm and controllable. The three reduction methods for the main model are evaluated for each of the three; the modified reduction is used for the corresponding cycle by itself. The professional reproductive technique for general straight frames decreases measurement time by approximately 200 for both versions. Are you concerned with the controllable damping strategy For the second highquest model, computational speeds up to three major degrees compared with conventional methodology are calculated according to the consolidating effects of re-enactment efficiencies and the study of eyewitness/control technologies based upon lowdemand models. There has been evidence of the output preparation of controllable process damping procedures, substantial acceleration in the re-launch, misuse of the restricting idea of non-linearity, and of the prospect of integrating decreased demand for audiences and input benefits into this worldview.

[9] The authors of this paper studied the nonlinear, rocking seismic response of slender independent columns, given they were fitted with verical energy dispersion devices along their sides (or at their pivot points). These devices provide either hysteretic or viscous (linear or non-linear). Nicos Makris, M.ASCE, and Mehrdad Aghagholizadeh. This paper derived nonlinear movement equations and then analyzed the South Rangitikei Rail Bridge's transverse reaction. It is evident in the study that, while reported ground movements are much stronger than the documented, powerful motions during the bridge construction, the 72-meter phase bridging pier exhibit remarkable seismic performance. Following that the study revealed that individual cases of earthquake excitations are more worsening than the undamped response, in which the rocking bridge jet reactions are damped with both hystereic and viscous dampers.
This can also arise when quantitative methods are idealized. The article concluded that the effectiveness of more hysteretic or viscous damping to avoid rocking reactions is primarily dependent on the cinematic properties of soil motion at a local level. If the damp reaction exceeds the undamped reaction, the degree is reduced and the damp reaction is, in most situations, smaller than the damp reaction.

[10] E. I. Barbato, I. Tubaldi, M. ASCE, A. Dall'Asta:This paper introduces a reliability-based manufacturing technique for seismic design in both freely and coupled configurations of the gooey / viscoelastic disipation units. This is a reliable approach to existing seismic design schemes focused on implementation, which specifically takes into account seismic data, model boundaries and the relations between the various cut-off states. The method suggested projects the challenge of the damping strategy for objective application as a reliability rationalising question with an imperative of probabilism. In specific, the complete approach suggested in this analysis was stochastic seismic stimulation and output thresholds for which the underlying behaviour could be regarded as specifically flexible. The rationalization dilemma is well solved under these circumstances, by using current logical methods to determine the efficacy of the system. This scientific plan is an approximation of the optimal plan that should be used in order to create an ideal plan as a starting point for recreation strategies. For the procurement of an updated plan scheme that is usually sufficiently similar to the ideal plan scheme resulting from fundamentally more expensive reproduction policies, an important revision equation was proposed. By considering all steel frameworks as straight, scalable different opportunities system for different direct damping features and the co-location, both free and coupling, the proposed plan solution was outlined and accepted. [11] James Newell, etc.:-The writers consulted the Viscous Wall Dampers hospital in San Francisco, 
California. In order to meet the strict performance expectations, VWDs have been selected for the paper survey as providing a reliable, maintenance-free, economic protection system. Further damping decreases the need for acceleration, reduces nonstructural bracing costs and reduces the potential non-structural damage after the earthquake. Fullscale VWD Prototype Testing was conducted to assess wind and seismic performance in accordance with the requirements of ASCE 7-05 and to assess appropriate features for a non-linear history of reaction. The VWDs are modeled on a viscous bar segment composed of fluid dampers and linear bars. A analytical methodology considering the upper and lower limiting damper effects has been implemented to take account of the expected continuum of building reaction resulting from potential heterogeneity of VWD properties. The results suggest that the seismotic building strength of the non-structural section of the roof over the other floors was reduced around 30\%, which reduced the total expense of nonstructural bracing.

[12] Zheng LU1; Kun Li; and Ying ZhOu:-Research and the development of a new kind of Particle damper considered to be the tuned mass damper, but with different damping mechanisms, with identical configuration and application structures. Systematic comparative analyses of such dampers are also particularly important for future implementations. This paper uses three examples, including a singledegree autonomy device, five-storeyed linear-elastical steel frame and a 20-story house for non- linear comparisons, to compare structural efficiency with an optimized tonic mass dump and an optimal specific stick. In optimal parameters of the particle damper, a separate evolution algorithm is used, and optimal parameters of the tuned mass damper are established for the same extra mass according to the classic Den Hartog principles. The numerical simulation shows that the correctly constructed partition damper has a stronger vibration management effect than the ideally calibrated mass damper: elastic efficiency and nonlinear performance indexes, number and maximum plastic hinge rotation as well as component energy dissipation. The most noticeable advantage of contrast is the reduction of the relative change and efficiency of the primary structure and of the damper. [13] Zheng Lu; Biao Huang; Zixin Wang; Ying Zhou:Curves the dynamic behavior of particle damper (PD) and Tuned Mass Damper (TMD) structures using enormous table experiments and aeroelastic tube checking. Parameters such as seismic waves, top land rates, damping ratios, mass ratios and attack angles are measured. A detailed study of the PD using the TMD shows that the vibrational control effects of $\mathrm{PD}$ typically are greater than those of TMD under both earthquake and wind charges. This is because PD dissipates the input energy through the impact and friction of particles between and between particles and tuning frequency parts (similar to TM). The experimental study of a single-grade independence (SDOF) unit with full PD and TMD and the concealed vibration control system also performed numerical calculations. The results show that the vibration regulation effects of both dampers are identical if the fluctuating amplitude of the added PD mass is much smaller than the TMD. Studies found the characteristics of the seismic input with different parameters and the wind angle of attack had a greater influence than the PD.

[14] Romanbabu. M. Romanbabu. Oinam and Dipti Ranjan Sahoo:-In the report, authoring the low seismic gravity reaction for reinforced concrete structures has been reduced by metal dampers. This research illustrates a strengthening strategy for a soft tale using a mixture of metallic yielding devices for the RC structure. In order to research his seismic response in various amplitudes of the chosen ground motion, a two-story, large-scale measuring test was performed pseudodynamically. A quasistatic cyclic measure for the resistance to failure of the improved specimen. In order to forecast the seismic response of 
the respectively enhanced CR structure, a numerical model is further developed using Free Sees machine softws. Along with the nonlinear pushover time dynamic experiments, the seismic reaction and the enhanced specimen resistance to collapse are expected. The findings of the numerical analysis were very consistent with the experimental results.

[15] In this paper Seismics' unwaving, quality-driven boundary radiation is always applied via a critical plan plot, which conjures up the Hrovat calculation with complex comparison power, and the plan for MR dämpfers for semi-active structural control. This analysis is based on the PSO control standard and the isolated observable minute strategy for preferably semi-active structure controls in previous work. An arbitrary, one-story building with an MR damper is discussed for illustrative purposes. Relative studies have been completed into probabilistic steps, plans and prices. For approval purposes, a six story building confined by MR dampers was investigated. A continuous semi-active quality control offers a better and more useful primary mechanism than a semiactive ideal observable minutes control. The critical plan includes a stronger interstory float, an almost comparative story of increasing speed regulation, and a marginally higher MR damping capacity than a stand-alone plan, which guarantees a proper cost/suitability balance. Semiactive stochastic ideal controls absorb the elegance of comparative control viability, using the accompanying, unequal configurations of the network that weights the weight. The semi-active stochastic optimum control achieve an appropriate unchanging primary output in global sense through the goodness of seismic reliability which is based on an integral plan and forces the smooth performance on a level of history.

[16] Suresh Lekshmi and K. [16]. M. Small scale:-This analysis investigated the feasibility as a non-inclusive vibration management technique of the different TMD frames. The application of the MTMD systems was tested for consonant and earthquake excitations.
Tentatively and systematically, a triple opportunities contour featuring single, double and triple TMDs was focussed on the effect symphony on structures. In order to understand the consequences of seismic excitement on infrastructure, ve-level opportunities system of single and double TMMDs was dismantled. By analyzing the framework responses in various architectures, the location of the best performing dampers was determined. The result revealed that the number of TMDs (tuned to the following higher normal frequencies) with pinnacle reactions decreased. Extension of the TMD quantity (tuned to the following higher frequencies) gives a higher transmission vibration control. The judgment about location of TMD is influenced by the abundance of the modal form associated with the repeat of the TMD tuning and, if it is put on the floor with the greatest fitness as a fiddle, the TMD strategy produces the maximum decline in pinnacles.

[17] Lekshmi Suresh and K, both. M. Mini:-These paper tuned TVMD and tuned Inert Dampers are tuned inert dampers (TIBDs), which are capable of use of amputated apparent weight or tuning effects. TVMD's are tuned inert-base dampers. This research explored the use of several TIBDs in order to minimize the seismic reactions of MDOFs. Here, TIBD settings and modes have been studied by defining an H2-based placement index. The TVMDs and TIDs have spread through the structure and are tuned into many modes in order to maximize the output of objective responses. The TVMD and TID are equivalently stiff and damped to reveal the mechanisms for TIBD regulation, such as essential additional damping of the resonance frequency. Earthquakes with varying spectral properties to confirm the efficacity of TIBD systems, for example, have been replicated in three and ten-story buildings. Results demonstrated a considerable reduction of root middle-square (RMS) displacement and speed reactions and higher than viscously chosen dampings 
and viscous damping in dispersed TIBDs in order to decrease peak displacement in the right mode.

[18] Luyu Li1 and Yongjia Du:- This analysis demonstrated that the TMD designed by a traditional TMD plan strategy that doesn't see the non-linerarity of TMD is not ideal for TMD, the consistent rooting sufficiency of solitary degree of optionality under TMD control by means of a consonant balancing technique (solidifying nonlinearity or relaxing nonlinearity). The left pinnacle of the system reoccurrence reaction bend is stronger in the state of solidificating nonlinearity, while the right pinnacle of the structure's re-occurrence bend is higher in the state of mellowing nonlinearity. Given the constraint of the counting cycle, the enhanced plan methodology contains significant errors, if the mass proportion is immense or if there is no separate structure for the TMD recurrence reaction bending. The optimal recurrence of the TMD is obtained by making two pinnacles of the structure recurrence reaction equal bend, improving the statistical equation by arranging the symphonic equilibrium technique and demonstrates high accuracy under the states of nonlinear intensity both solidifying and molten. In addition, the use of this technical method can better deal with the case of high-left or rightpinnacle evaluations and can boost the management influence of TMDs when recognizing nonlinearity of customary strategy technique. In addition, the method suggested would be useful to control the vibration of actual structures. If TMD gadget is used for control purposes, the key vibration of massive structures, especially large-scale, will illustrate nonlinear practices due to enormous defigurations. The suggested approach should, in this case, understand the contrasting superior control effect and customary plan techniques.

[19] John R. Sladek, Richard E. Klingner:-The installation of Tuned Mass Dampers (TMD) to minimize the wind response in high-rise buildings such as John Hancock Building in Boston or Citicorp
Centre, New York City have recently been identified in various Engineering Publications (6,7,9,10). In both systems, TMDs were built to alleviate occupants' pain $(10,11)$ and wind accelerations were decreased up to 40 percent (10). The aim of this thesis is to explore whether TMD or related instruments can or could not be utilized to minimize structures' response to earthquake earthquakes (checked)

[20] The researchers in this paper researched the tuned vibratory vibration retention portion of fluids and Swaroop K SwarOop K. Yalla, student society member and Ahsan Kareem (TLCDs). TLCD's are a group of tuned fluid dampers that provide roundabout damping of the critical structure by shifting the liquid section into a $\mathrm{U}$-formed compartment. The dispersion of energy in the water portion results from fluid input into a vacuum with intrinsic headaches. Damping in a TLCD typically depends on a head-to-head portion, fluid waving speed, and is not linear because of its 4faceted term. Another technology has also been developed to depart from the TLCD and the Analog Linearising Plot for the optimum typical coefficient of a certain degree of wind or seismic excitement in one advance. Introduction of a band of sifted source sound to twist sismic loads The highest damping and tuning ratio for a TLCD using a solo chance map, with a reproducible tone. The main damping advantages have also been explored in several TLCDs. Multiple TLCDs are provided as a model for tracking different wind excitation modes.

\section{CONCLUSION}

1. The MTLCD configuration makes an acceptable portability in different areas, simple to mount, with the limited size of each TLCD (Tuned Live Column Dampers).[20].

2. The modal frequency has increased higher modal damping ratios and it has proven that a straight variance is superior to that commonly found in 
proportion, rigidity or Rayleigh damping model .[5]

3. MTMD is suitable for the management of dynamism in large buildings under wind forces as opposed to STMD Multiple Mass dampers (Single Tuned Mass Damper). There was a mistake .[6]

4. Diagram of rock reactions and concluded that the ability for more hysteretic or viscous damping in rocking reaction suppression depends significantly on the local filming characteristics of ground movements.

5. An simple, but effective technique to build and to position viscous and viscoelastic dissipative devices in linear elastic structural structures during seismic testing. There was a mistake .[10]

6. The use of external damping decreases earthquake demand, reduces non-structural expense for bracing and reduces potential losses following the earthquake..... There was a mistake .[11]

7. In PD (Particle Damper), it is more stable than in TMD (Tuned Mass Damper) .[11]

\section{REFERENCES}

[1]. Non-structural Component Seismic Spectral Accelerations in Two Existing Steel Buildings with Supplemental Damping Kenneth O'Neill, P.E.; Erik Bishop, P.E. ; David Swanson, P.E., S.E. ; Bradley Martin, P.E., S.E. ; and Drew Nielson.-2017

[2]. Research on Differences between Cylindrical and E-Shaped Dampers for the Bidirectional Seismic Control Hao Gaol and Junjie Wang, Ph.D.-2020

[3]. Optimal Evolutionary Seismic Design of ThreeDimensional Multistory Structures with Damping Devices Georgios Apostolakis, A.M.ASCE-2020
[4]. Seismic Retrofit of Structures Using Hybrid Steel Slit-Viscoelastic Dampers; Mohammad Seddiq Eskandari Nasab and Jinkoo Kim-2020

[5]. Evaluation of Damping Ratios for the Seismic Analysis of Tall Buildings Cristian Cruz1 and Eduardo Miranda, Ph.D-2018

[6]. Wind Response Control of Tall Buildings with Flexible Foundation using Tuned Mass Dampers Said Elias* Vasant Matsagar-2018

[7]. Effects of Hysteretic Properties of Stud-Type Dampers on Seismic Performance of Steel Moment-Resisting Frame Buildings Po-Chien Hsiao1 and Wei-Chieh Liao-2019

[8]. Rapid Controllable Damper Design for Complex Structures with a Hybrid Reduced-Order Modeling/Simulation Approach Mahmoud Kamalzare ; Erik A. Johnson, M.ASCE ; and Steven F. Wojtkiewicz, M.ASCE-2015

[9]. Effect of Supplemental Hysteretic and Viscous Damping on Rocking Response of FreeStanding Columns Nicos Makris, M.ASCE ; and Mehrdad Aghagholizadeh, S.M.ASCE-2019

[10]. Efficient Approach for the Reliability-Based Design of Linear Damping Devices for Seismic Protection of Buildings ; E. Tubaldi ; M. Barbato, M.ASCE ; and A. Dall'Asta - 2015

[11]. Seismic Design of a 15 Story Hospital Using Viscous Wall Dampers ; James Newell, Ph.D., P.E. , Jay Love, S.E. , Mark Sinclair, S.E., YuNing Chen, P.E. , and Amarnath Kasalanati, Ph.D., P.E.-2011

[12]. Comparative Studies on Structures with a Tuned Mass Damper and a Particle Damper; Zheng Lu ; Kun Li ; and Ying Zhou, M.ASCE2018

[13]. Experimental Comparison of Dynamic Behavior of Structures with a Particle Damper and a Tuned Mass Damper; Zheng Lu; Biao Huang ; Zixin Wang ; and Ying Zhou, M.ASCE -2018

[14]. Using Metallic Dampers to Improve Seismic Performance of Soft-Story RC Frames: 
Experimental and Numerical Study;

Romanbabu M. Oinam, Ph.D.1 ; and Dipti Ranjan Sahoo, Ph.D-2018

[15]. Optimal MR Damper-Based Semiactive Control Scheme for Strengthening Seismic Capacity and Structural Reliability; Yongbo Peng and Zhenkai Zhang-2020

[16]. Effect of Multiple Tuned Mass Dampers for Vibration Control in High-Rise Buildings Lekshmi Suresh1 and K. M. Mini, Ph.D-2019

[17]. Design and Evaluation of Tuned Inerter-Based Dampers for the Seismic Control of MDOF Structures ; Yongkui Wen ; Zhengqing Chen, M.ASCE ; and Xugang Hua-2016

[18]. Design of Nonlinear Tuned Mass Damper by Using the Harmonic Balance Method Luyu Li and Yongjia $\mathrm{Du}-2020$

[19]. Design of Nonlinear Tuned Mass Damper by Using the Harmonic Balance Method ; Luyu Li1 and Yongjia Du

[20]. Optimum absorber parameters for Tuned Liquid Column Dampers ; Swaroop K. Yalla,1 Student Member, ASCE, and Ahsan Kareem,2 Member, ASCE

\section{Cite this article as :}

Avinash Kalamkar, N. H. Pitale, P. B. Patil, "Use of Added Damping Devices with Special Emphasis on Multiple Tuned Mass Dampers : A Review", International Journal of Scientific Research in Science and Technology (IJSRST), Online ISSN : 2395-602X, Print ISSN : 2395-6011, Volume 8 Issue 1, pp. 227-235, January-February 2021. Available at doi : https://doi.org/10.32628/IJSRST218147

Journal URL : https://ijsrst.com/IJSRST218147 